\title{
A Design Method for Mode-Selective Waveguide Couplers in Dual-Polarized Wideband Matched-Feed Antennas
}

Palvig, Michael Forum; Meincke, Peter; Jørgensen, Erik; Breinbjerg, Olav

Published in:

I E E E Transactions on Antennas and Propagation

Link to article, DOI:

10.1109/TAP.2017.2781252

Publication date:

2017

Document Version

Peer reviewed version

Link back to DTU Orbit

Citation (APA):

Palvig, M. F., Meincke, P., Jørgensen, E., \& Breinbjerg, O. (2017). A Design Method for Mode-Selective Waveguide Couplers in Dual-Polarized Wideband Matched-Feed Antennas. I E E E Transactions on Antennas and Propagation, 66(2), 990 - 995. https://doi.org/10.1109/TAP.2017.2781252

\section{General rights}

Copyright and moral rights for the publications made accessible in the public portal are retained by the authors and/or other copyright owners and it is a condition of accessing publications that users recognise and abide by the legal requirements associated with these rights.

- Users may download and print one copy of any publication from the public portal for the purpose of private study or research.

- You may not further distribute the material or use it for any profit-making activity or commercial gain

- You may freely distribute the URL identifying the publication in the public portal 


\title{
A Design Method for Mode-Selective Waveguide Couplers in Dual-Polarized Wideband Matched-Feed Antennas
}

\author{
Michael Forum Palvig, Student Member, IEEE, Peter Meincke, Member, IEEE, Erik Jørgensen, Member, IEEE, \\ Olav Breinbjerg, Senior Member, IEEE
}

\begin{abstract}
A novel type of multihole directional coupler is presented as well as a design method involving novel procedures. The device couples two orthogonal $\mathbf{T E}_{11}$ modes in a circular waveguide into two orthogonal $\mathbf{T E}_{21}$ modes with specified excitations in a surrounding coaxial waveguide, which has the circular waveguide as inner conductor. A simple, novel analytical procedure is used to determine azimuthal hole positions, such that each of the input modes couples to only the desired coaxial $\mathbf{T E}_{21}$ mode. The method is applied to a specific coupler which is designed to couple $-14 \mathrm{~dB}$ to both higher-order coaxial modes in the band from $10 \mathrm{GHz}$ to $14 \mathrm{GHz}$. The suppression of undesired modes is generally better than $40 \mathrm{~dB}$. The coupler is intended for broadband, dual-polarized matched feeds, which are able to compensate the intrinsically high cross polarization of offset single reflector antenna systems.
\end{abstract}

Index Terms-Directional Coupler, Reflector antenna feeds, Coaxial aperture antennas, Cross polarization

\section{INTRODUCTION}

Parabolic reflectors are the preferred choice for high gain antenna systems. Often, the reflector is chosen to be offset from the feed or subreflector in order to eliminate blockage. A drawback of the offset system is that the asymmetry of the configuration introduces high cross polarization. For dual reflector systems, this can be compensated by applying the Mizuguchi condition [1]. For a single reflector system, an alternative solution was proposed by Rudge and Adatia in 1975 [2]. It involves letting cross polarization from the feed antenna compensate the cross polarization introduced by the offset geometry. This is achieved by generating higher-order modes in a feed horn antenna that have specific amplitude and phase relationships with the fundamental mode. Such a feed is often called a Matched Feed. For a circular horn, the proposed higher-order mode was $\mathrm{TE}_{21}$ in two orthogonal rotations for two operating polarizations.

Matched feeds have received considerable attention in recent years. Feed designs with circular [3]-[6], rectangular [7], [8], corrugated [9]-[11], and other geometries [12], [13] have been presented. The primary challenge of the matched-feed technique is to achieve a practically sufficient bandwidth for

O. Breinbjerg is with the Department of Electrical Engineering, Electromagnetic Systems, Technical University of Denmark, DK-2800 Kgs. Lyngby, Denmark. (email: ob@elektro.dtu.dk)

E. Jørgensen, and P. Meincke are with TICRA Engineering Consultants, DK-1119 Copenhagen K, Denmark. (email: ticra@ticra.com)

M. F. Palvig is affiliated with both of the above institutions. (email: mfp@ticra.com)

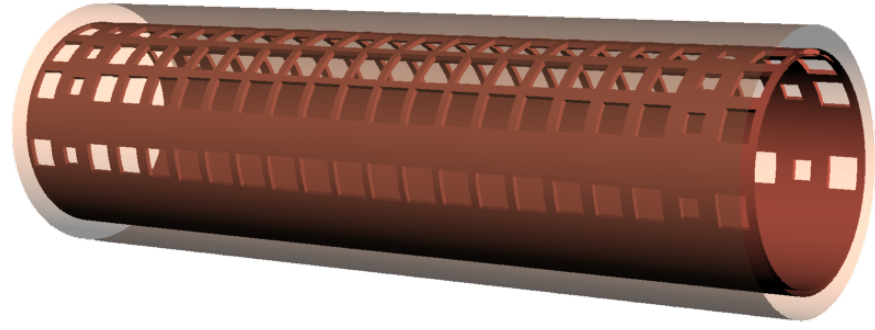

Fig. 1. Hole distribution in the coupler. The outer conductor of the coaxial part has been made transparent such that the holes are visible. The square hole sizes are from either end: $2.86,1.77,2.79,2.74,2.01,3.09,3.14,3.16$, $3.30,3.33$, and $3.27 \mathrm{~mm}$.

dual polarization applications, which is essential for the use of this technology in communication applications. This difficulty stems from the fact that the fundamental and higher-order modes have different cutoff frequencies, resulting in phase dispersion. The bandwidth can be defined as the frequency range within which the peak cross polarization is reduced by a certain amount, e.g. by $10 \mathrm{~dB}$ relative to the case with a purely co-polarized feed. Watson et al. [14] were first to introduce this bandwidth measure, achieving $4 \% 10 \mathrm{~dB}$-bandwidth for both polarizations. By using an optimization procedure and trading maximum reduction for more bandwidth, the authors achieved a $7 \mathrm{~dB}$ reduction over a $12 \%$ bandwidth for both polarizations [15]. Using a novel mode launching concept, Dey et al. [16] achieved a $10 \mathrm{~dB}$ reduction over a $10 \%$ bandwidth for a single polarization.

A way to mitigate the phase dispersion problem is to somehow use higher-order modes that have the same or nearly the same cutoff frequency as the fundamental mode. This is not possible for conventional waveguide geometries, except if the waveguide walls are anisotropic impedance surfaces [17]. Jana and Bhattacharjee [13] presented an optimized waveguide cross section which brings the cutoffs closer together. Pour and Shafai [12] proposed coupling the higher-order modes to a coaxial waveguide surrounding the primary one. The higherorder modes are then radiated directly from the coaxial guide as in [18]. It turns out that the dimensions can be engineered such that the cutoff of the $\mathrm{TE}_{21}$ mode in the coaxial waveguide is close to that of the $\mathrm{TE}_{11}$ mode in the circular waveguide. In [12] the bandwidth is limited because of the resonance of a single coupling hole and the design is single polarized.

The present work is based on the concept in [12], but 
is extended to a multi-hole design in order to support dual polarizations and avoid resonances of individual holes. The device is designed as a distributed directional waveguide coupler, which has not previously been done for matchedfeed mode launchers. The presented design method concerns the mode coupler itself, not the full antenna. The theoretical basis of coupling between waveguides through small holes was laid by Bethe [19] in 1944. Later, Miller [20] described the theory of distributed couplers with tapered coupling functions to enhance directivity and discrimination of undesired modes. This theory was utilized in [21], [22] to create mode selective directional couplers, coupling specific higher-order modes from a circular waveguide into rectangular ones. This work follows the same methodology, but is extended to account for unequal cutoff of input and output modes, and for the fact that the two output modes are not isolated in rectangular waveguides, but must coexist in the same coaxial waveguide. The latter challenge is overcome by a simple analytical method for determining an azimuthal hole distribution (in addition to the longitudinal one) which enables coupling from two orthogonal input modes to two orthogonal output modes, without cross coupling the wrong modes. To the authors' knowledge, this type of directional coupler and elements of the design method are not previously published in the literature.

The paper is organized as follows: In Section II, we shall briefly rederive the loose coupling theory of Miller [20] followed by determination of optimal coupling coefficients in Section III. Then we will address the optimal selection of the azimuthal distribution of coupling holes in Section IV. Then, by simulation experiments, Section $\mathrm{V}$ will cover optimal hole shape and simple, but effective mapping from coupling coefficients to hole sizes. Finally, the resulting design will be analyzed by full-wave simulation in Section VI.

\section{LoOse Coupling TheORY}

Loose coupling theory, as opposed to tight coupling theory, assumes that the wave in the driven line is unpertubed by the coupling [20]. For matched feeds, we are interested in coupling only a small amount of the power to the coaxial waveguide, and a tight coupling analysis can thus be omitted. The theory is quite intuitive and can be written up by inspection. For the use in subsequent sections, we briefly rederive loose coupling for couplers with discrete coupling points. Note that the definitions differ slightly from [20].

Consider a wave incident on a transmission line, the input line. The wave is coupled to another line, the output line, at discrete coupling points over a section of length $L$, see Fig. 2. The phase constant in the input line is $\beta_{\text {in }}$ and the phase constant in the output line is $\beta_{\text {out }}$. If the lines are modes in smooth walled waveguides, the phase constant $\beta$ of each line is found from the cutoff frequency as

$$
\beta=\frac{2 \pi}{c} \sqrt{f^{2}-f_{c}^{2}},
$$

in which $c$ is the speed of light, $f$ is the frequency, and $f_{c}$ is the cutoff frequency. At each coupling point, the complex coupling coefficient, $a_{m}$, determines the amplitude and phase of the coupled wave relative to the input wave at the coupling location. The input and output line can represent modes in different waveguides or two modes in the same waveguide.

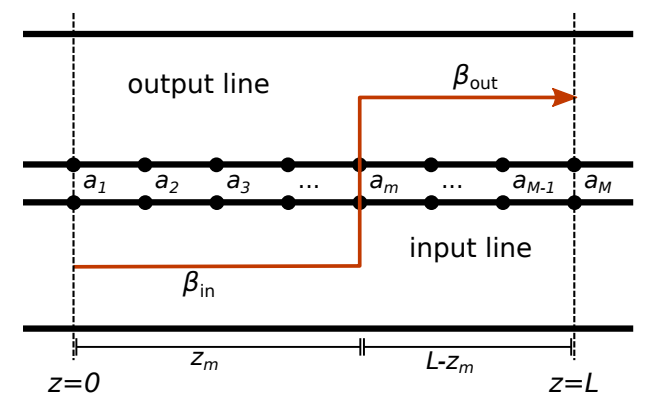

Fig. 2. Loose coupling with discrete coupling points.

The contribution to the coupled wave in the forward direction at $z=L$ from a single hole at $z=z_{m}$, as depicted in Fig. 2, can be expressed as

$$
\begin{aligned}
I_{f m} & =e^{-j \beta_{\mathrm{in}} z_{m}} a_{m} e^{-j \beta_{\text {out }}\left(L-z_{m}\right)} \\
& =a_{m} e^{-j \beta_{\text {out }} L} e^{-j z_{m}\left(\beta_{\text {in }}-\beta_{\text {out }}\right)},
\end{aligned}
$$

in which a timefactor of $e^{j \omega t}$ is used. Summing over all coupling points yields the total forward coupled wave

$$
I_{f}=e^{-j \beta_{\text {out }} L} \sum_{m=1}^{M} a_{m} e^{-j z_{m}\left(\beta_{\text {in }}-\beta_{\text {out }}\right)} .
$$

Similarly, the contribution of the hole to the coupled wave in the backward direction at $z=0$ in the output line is

$$
\begin{aligned}
I_{b m} & =e^{-j \beta_{\text {in }} z_{m}} a_{m} e^{-j \beta_{\text {out }} z_{m}} \\
& =a_{m} e^{-j z_{m}\left(\beta_{\text {in }}+\beta_{\text {out }}\right)}
\end{aligned}
$$

and the total backward coupled wave thus

$$
I_{b}=\sum_{m=1}^{M} a_{m} e^{-j z_{m}\left(\beta_{\mathrm{in}}+\beta_{\mathrm{out}}\right)} .
$$

Note that both the backward and forward coupled waves can be evaluated from the function

$$
F(\theta)=\sum_{m=1}^{M} a_{m} e^{-j z_{m} \theta}
$$

where $\theta=\beta_{\text {in }}-\beta_{\text {out }}$ and $\theta=\beta_{\text {in }}+\beta_{\text {out }}$ for forward and backward coupling, respectively. It is seen that $F(\theta)$ is simply a Fourier transform of the coupling distribution in the spectral variable $\theta$, transforming the coupling coefficients to the $\theta$ domain. If the coupling coefficients, $a_{m}$, are symmetric and all have the phase $\angle a$, it can be shown that the phase difference between the output and input wave at the end of the coupler is

$$
\Delta \phi=\angle a+\frac{L}{2}\left(\beta_{\text {in }}-\beta_{\text {out }}\right) .
$$

If the cutoffs of the input and desired coupled wave are identical, the phase constants are the same, and $\theta$ for the forward direction is zero for all frequencies. This condition is sought in most traditional directional couplers. Then the function $F$ is designed to have the desired coupling value at $\theta=0$ and a lower value in $\theta$-ranges of undesired modes, 
backward and forward [20]. In our case, the cutoff of the desired mode in the coaxial waveguide is close to that of the input mode, but may not be exactly equal, so we cannot assume that $\theta$ is zero.

\section{Design of Optimal Directional Coupler}

We will find the optimal coefficients, $a_{m}$, given certain criteria. The length of the coupler and number of coupling points are first to be determined. This choice corresponds to choosing filter length and sampling frequency of a digital Finite Impulse Response (FIR) filter. Among other things, it determines the steepness of the filter roll-off in the spectral domain. For the present design there are additional considerations to take into account: 1) we want the coupler as short as possible, 2) holes must not overlap, and 3) coefficient values must be within a feasible range. Balancing these goals, we arrive at a coupler length of $80 \mathrm{~mm}$ with 21 holes. The length of the coupler is measured from the center of the first hole to the center of the last, and the remaining holes are equally distributed in between.

We consider a circular waveguide surrounded by a coaxial waveguide such that the circular waveguide constitutes the inner conductor of the coaxial one. The principal dimensions are listed in Table I. We are interested in coupling orthogonal $\mathrm{TE}_{11}$ modes in the circular guide to orthogonal $\mathrm{TE}_{21}$ modes in the coaxial guide as shown in Fig. 3. The outer radius is chosen to approximately fulfill $a_{\text {outer }}=1.173 a_{\text {circ }}-a_{\text {inner }}$, which ensures that the cutoff of $\mathrm{TE}_{21}$ in the coaxial guide is nearly equal to the cutoff of $\mathrm{TE}_{11}$ in the circular guide [23, p. 70,77] (see Table I).

For the present design, the power of the desired coupled mode compared to the input power should be $-14 \mathrm{~dB}$, which is approximately the amount needed to compensate the cross polarization in an offset reflector system with a focal length to diameter ratio (f/D) of 0.6. The frequency band of operation is $10 \mathrm{GHz}$ to $14 \mathrm{GHz}$, i.e. a fractional bandwidth (FBW) of $33 \%$.

The $\theta$-values for the forward and backward coupling of each mode can now be found from the above. Mapping the operating frequency range to $\theta$-values results in Fig. 4. A range of $\theta$-values are identified as undesired, which we will denote the stopband in the $\theta$-domain. The passband given by the green line is comparatively narrow owing to the closeness in cutoff between input and desired coupled modes (see Table I).

TABLE I

PRINCIPAL DIMENSIONS AND CUTOFF FREQUENCIES OF THE COUPLER

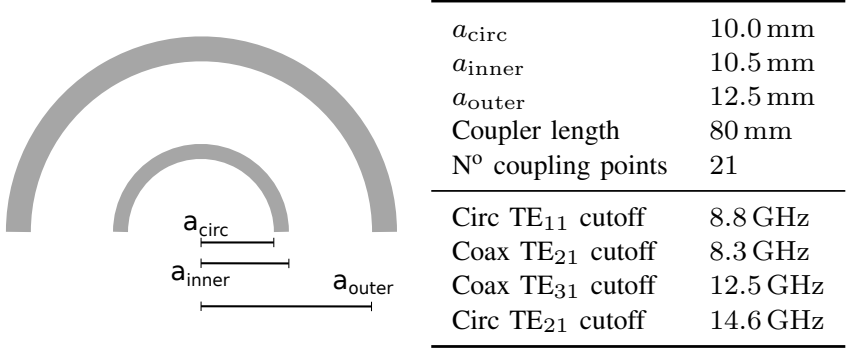

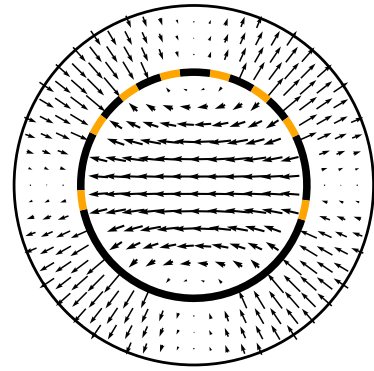

(a)

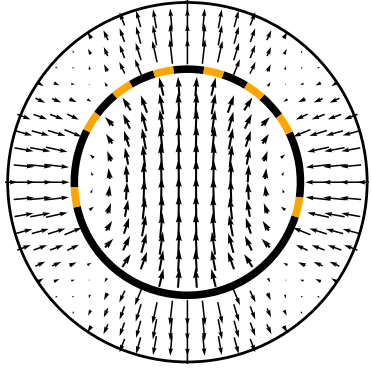

(b)
Fig. 3. Modal E-fields of the incident circular and desired coaxial modes for the two polarizations: (a) $\mathrm{TE}_{11}^{1}$ to $\mathrm{TE}_{21}^{2}$ and (b) $\mathrm{TE}_{11}^{2}$ to $\mathrm{TE}_{21}^{1}$. The coupling holes are indicated in orange and their angular positions in degrees are $\phi=\left\{30.9^{\circ}, 53.8^{\circ}, 76.7^{\circ}, 102.1^{\circ}, 125.0^{\circ}, 147.9^{\circ}, 187.6^{\circ}, 347.4^{\circ}\right\}$.

The coupling function (6) is equivalent to the frequency response of a FIR filter with filter coefficients $a_{m}$. The coupler can thus be designed as a FIR filter, defining stop- and passbands in the spectral $\theta$-domain. Optimal coefficients can be determined by standard techniques for FIR filter design. The coefficients in Fig. 5 are found from the SciPy [24] implementation of [25], which minimizes the maximum deviation from the desired coupling in each band. The coupling holes will all be electrically small and reactive, yielding coupling coefficients with constant phase, $\angle a$, close to $90^{\circ}$. This phase relationship is desirable for matched feed design. From Eq. (6), the resulting $\theta$-response is shown in Fig. 6. Passand stopbands are indicated in green and red, respectively.

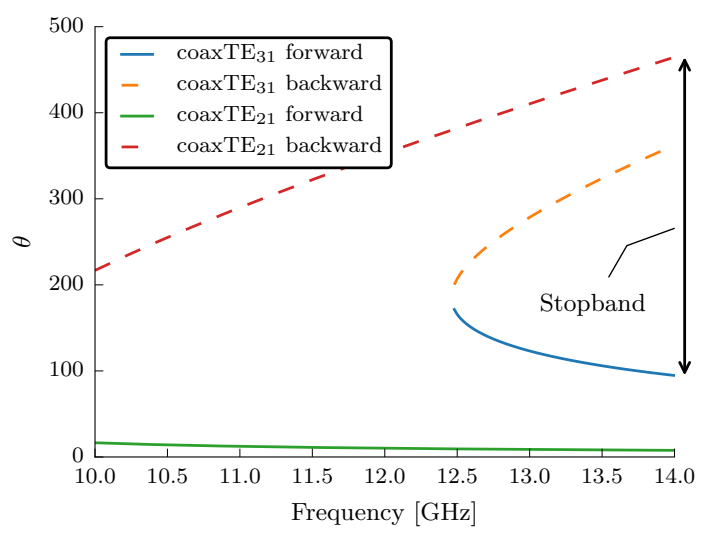

Fig. 4. $\theta$-values evaluated over the frequency band of interest in order to find pass- and stop bands in the $\theta$-domain.

\section{Azimuthal Hole Distribution}

As explained in the previous section, the distribution of holes in the longitudinal direction can suppress the generation of undesired modes with different phase constants than the desired, but it cannot distinguish modes with the same phase constant. Since we require that each polarization of $\mathrm{TE}_{11}$ couples only to the correct $\mathrm{TE}_{21}$ mode, unwanted $\mathrm{TE}_{21}$ couplings must be suppressed in another way. We denote two orthogonal versions of the circular modes with superscripts 1 and 2: $\mathrm{TE}_{11}^{1}$ should couple to $\mathrm{TE}_{21}^{2}$, but not to $\mathrm{TE}_{21}^{1}$ and vice versa as depicted in Fig. 3. The suppression is achieved by placing 


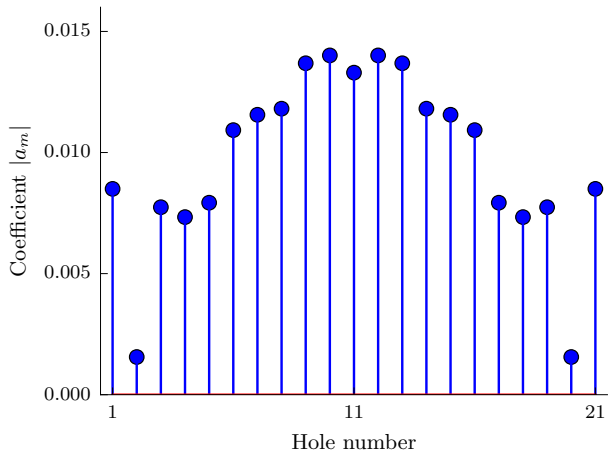

Fig. 5. Optimal coupling coefficients minimizing the maximum deviation from the desired coupling in both bands, i.e. $-14 \mathrm{~dB}$ in the passband and $-\infty \mathrm{dB}$ in the stopband. All coefficients have the same phase.

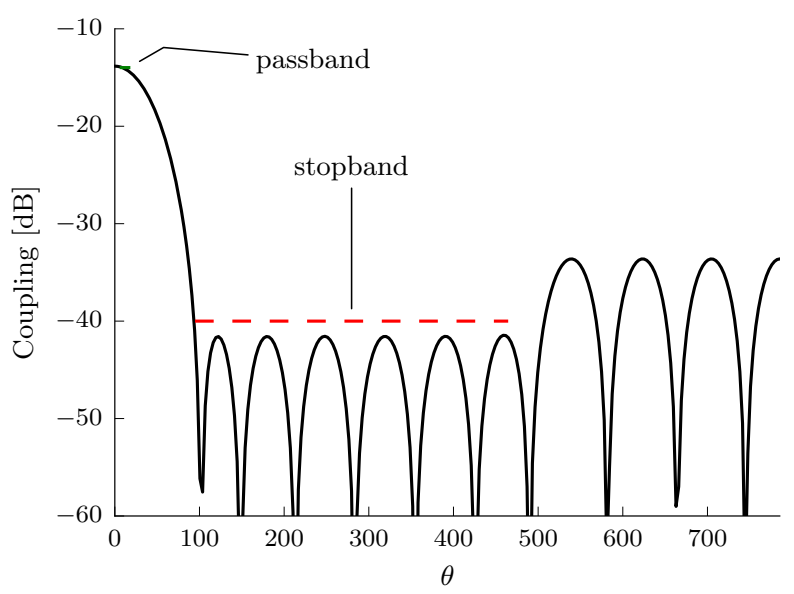

Fig. 6. $\theta$-response of the coupling coefficients. The $\theta$ ranges of desired and undesired modes are indicated by passband and stopband, respectively.

several holes around the circumference at each coupling point along $z$.

The coupling from one mode to another through a waveguide wall can be modelled by representing the hole with an electric dipole normal to the wall and a magnetic dipole parallel to the wall [19]. The dipoles are excited proportional to the respective input fields: normal E-field and tangential $\mathrm{H}$-field. The proportionality constants, the polarizabilities of the hole, depend on the hole shape and size. The dipoles radiate and excite modes in the output waveguide. The strength of each excited mode is proportional to that mode's field projected onto the dipoles [26, Sec. 4.10].

From these considerations, we can determine the effect of moving a coupling hole around the circumference of the circular waveguide wall. For circular and coaxial waveguide modes, the azimuthal $(\phi-)$ variation of the fields is given by sine and cosine functions of the $\phi$ variable times the azimuthal $(m-)$ index of the modes. The $\phi$-variation of a mode coupling is a product of the field variation for the input and output mode. The resulting $\phi$-dependence of the desired and the most critical undesired couplings are listed in Table II.

With this information, we can select a number of hole positions, such that the combined contributions from all holes will cancel out undesired couplings [27]. Additionally, the
TABLE II

SUMMARY OF DESIRED AND UNDESIRED MODE COUPLINGS

\begin{tabular}{cc}
\hline Coupling & $\phi$-dependence \\
\hline Desired & \\
$\mathrm{TE}_{11}^{1} \rightarrow \mathrm{TE}_{21}^{2}$ & $\cos \phi \sin 2 \phi$ \\
$\mathrm{TE}_{11}^{2} \rightarrow \mathrm{TE}_{21}^{1}$ & $\sin \phi \cos 2 \phi$ \\
Undesired & \\
$\mathrm{TE}_{11}^{1} \rightarrow \mathrm{TE}_{21}^{1}$ & $\cos \phi \cos 2 \phi$ \\
$\mathrm{TE}_{11}^{2} \rightarrow \mathrm{TE}_{21}^{2}$ & $\sin \phi \sin 2 \phi$ \\
$\mathrm{TE}_{11}^{x} \rightarrow \mathrm{TE}_{31}^{x}$ & $\cos \phi \cos 3 \phi$ \\
& $\cos \phi \sin 3 \phi$ \\
& $\sin \phi \sin 3 \phi$ \\
& $\sin \phi \cos 3 \phi$ \\
\hline
\end{tabular}

two desired couplings should be of approximately the same strength and the hole positions must be separated a certain distance such that they do not overlap for a certain hole size.

The process of choosing hole positions is automated with an optimization procedure. A local minimax optimizer (fminimax of Matlab) is used to minimize undesired couplings while satisfying constraints. Since the objective functions are computationally very light, the global best solution is sought simply by trying many starting guesses at random.

The resulting configuration is the 8 hole distribution indicated with orange in Fig. 3. This configuration suppresses undesired $\mathrm{TE}_{21}$ couplings by approximately $30 \mathrm{~dB}$ compared to desired ones. Undesired $\mathrm{TE}_{31}$ couplings are suppressed by $22 \mathrm{~dB}$, but these can be further suppressed by the arrangement of holes in the longitudinal direction as described in Sec. III. The coupler consists of 21 slices of equal thickness corresponding to the 21 coupling points. Each coupling point, or slice, will have 8 equally sized holes with the optimized $\phi$ distribution, but the hole size will change from slice to slice in correspondence with the coefficients in Fig. 5.

\section{MAPPing Coefficients to Hole Sizes}

The amount of coupling into each hole can be estimated by Bethe theory [19] or by improved methods [28], [29]. However, we shall take a shortcut and use a simpler, but very effective method. Since the coupling generally has a cubic relationship with the size of the hole [19], we just make a few simulation experiments with different hole sizes and fit a cubic spline to the data. In this way, effects like wall thickness and aperture shape are automatically taken into account. The simulations are carried out with a higher-order method of moments solver from TICRA [30]. Generalized scattering parameters between four waveguide ports are extracted: one circular and one coaxial at each end of the slice. The results are in excellent agreement with another commercial tool [31] (not shown here).

The holes are chosen to be square, in effect, there is a contribution to the coupling from both a longitudinal and an azimuthal equivalent magnetic dipole. This reduces the frequency dependence of the isolated hole, since the longitudinal coupling is inversely proportional to frequency and the azimuthal coupling is proportional to frequency [32]. 
Using the azimuthal hole distribution illustrated in Fig. 3, we simulate a single slice of the waveguide structure. The results are shown in Fig. 7. Six hole sizes are simulated and cubic splines are fitted to the data. Note that as a consequence of the optimized azimuthal hole distribution, the two desired couplings are nearly equal and the undesired $\mathrm{TE}_{21}$ couplings are suppressed. The data shown in Fig. 7 is averaged over the frequency range of interest: $10 \mathrm{GHz}$ to $14 \mathrm{GHz}$.

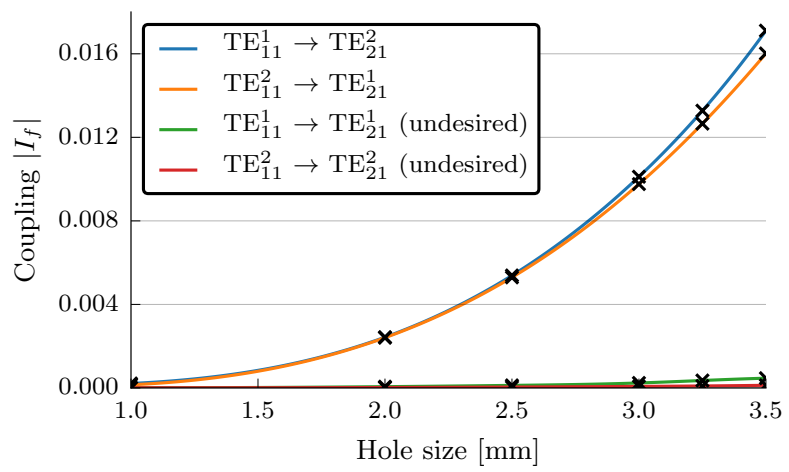

Fig. 7. Interpolation of the amount of desired coupling as a function of hole size. Based on six experiments indicated by black markers. The data for each hole size is averaged over a frequency range from $10 \mathrm{GHz}$ to $14 \mathrm{GHz}$. This data is used to map coupling coefficients to hole sizes. The undesired $\mathrm{TE}_{21}$ couplings are also shown, the suppression is better than $30 \mathrm{~dB}$ for the hole sizes used.

Using the interpolating function, all the coefficients of Fig. 5 are mapped to hole sizes. A coupler device with the resulting hole distribution is rendered in Fig. 1. In case there are any negative coupling coefficients, these can be implemented by negating the $\phi$-positions of the holes in the corresponding slice. This results in coupling of the opposite sign of both the desired coupling components (a consequence of the single sine factor in these couplings, Table II).

\section{COUPLER PERformance}

The performance of the coupler is evaluated by the same computation method as the single slice. Again, a coaxial and circular waveguide port is placed at each end of the coupler. The coaxial port at the back of the coupler will be terminated in a practical design, but is included in the simulation to ensure that very little power is coupled in the backward direction. As mentioned we are interested in having a constant coupling from $\mathrm{TE}_{11}^{1}$ to $\mathrm{TE}_{21}^{2}$ and from $\mathrm{TE}_{11}^{2}$ to $\mathrm{TE}_{21}^{1}$. All other possible couplings are desired to be low.

Several couplings (scattering parameters) are plotted in Fig. 8 as a function of frequency. The goal level of $-14 \mathrm{~dB}$ for the two desired couplings is indicated by a horizontal dashed black line. The realized coupling for both polarizations are within $2 \mathrm{~dB}$ of the desired over the entire band and within $0.5 \mathrm{~dB}$ of each other.

Undesired couplings are all the $\mathrm{TE}_{21}$ couplings in the backward direction, undesired $\mathrm{TE}_{21}$ couplings in the forward direction, and all $\mathrm{TE}_{31}$ couplings (see Table II). The level of the undesired couplings is below $-40 \mathrm{~dB}$ in the design band $10 \mathrm{GHz}$ to $14 \mathrm{GHz}$, except for one of the $\mathrm{TE}_{31}$ couplings. This coupling increases at higher frequencies to a level of about
$-36 \mathrm{~dB}$ at $14 \mathrm{GHz}$, which is still sufficient suppression for our application. All couplings to the backward port in the coaxial waveguide are low, and we can thus justify that terminating it will not affect performance. Also, the reflection at the input circular waveguide is of general interest, and is better than $-35 \mathrm{~dB}$ in the design band for both polarizations.

The reason for the increased $\mathrm{TE}_{31}$ coupling shown in green in Fig. 8, can be explained by the $\theta$-domain (Figs. 6 and 4): the $\theta$-values of the $\mathrm{TE}_{31}$ couplings come close to the passband at higher frequencies. From Fig. 6, it is evident that the filter response only just drops off quickly enough from passband to stopband. The filter design is based on phase constants of modes in the simple, unperforated waveguides. The many holes are likely to disturb the cutoff frequencies and phase constants of the modes in both the circular and coaxial guide, thus shifting the bands in the $\theta$-domain. This is most likely the reason for slightly higher $\mathrm{TE}_{31}$ coupling at the end of the band.

The relative phase of desired modes to the input mode is shown in the bottom part Fig. 8. The realized phase is close to the expected result from Eq. (7), which is obtained assuming $\angle a= \pm 90^{\circ}$. Choosing different waveguide dimensions can bring the expected - and realized - relative phases closer to $\pm 90^{\circ}$. However, lower values can be desirable to balance the opposite effect, if a subsequent horn section is added to the coupler.

The coupler presented here was designed with very little computational effort, from filter theory and analytical hole coupling theory. Only full wave simulation of a single slice was used in the design to establish the mapping from coefficients to hole sizes. Nevertheless, the resulting coupler performs well and as expected. Due to the nonresonant nature of coupling holes, the design is insensitive to manufacturing tolerances; this is supported by preliminary simulation experiments (not shown here).

\section{CONCLUSION}

A design method for a directional coupler coupling orthogonal $\mathrm{TE}_{11}$ modes from a circular waveguide into orthogonal $\mathrm{TE}_{21}$ modes in a coaxial waveguide surrounding the circular one, is presented. The method of mode selective directional couplers needed to be extended to allow coupling of specific degenerate modes into the same waveguide, where the coupled modes do not have the exact same cutoff as the source modes.

Optimal finite impulse response filter (FIR) techniques have been used in the determination of hole distribution in the longitudinal direction. This allows for discrimination of undesired modes which travel in the backward direction or have a different cutoff than desired modes.

Undesired $\mathrm{TE}_{21}$ modes which have the same cutoff as desired modes, cannot be suppressed by directional coupler theory. A novel method which optimizes the azimuthal distribution of the holes ensures that only the correct $\mathrm{TE}_{21}$ mode from each $\mathrm{TE}_{11}$ mode is coupled and that they both couple in equal amount.

The method is applied to a specific design which exhibits good performance over the band ( $33 \%$ FBW). Only a single 

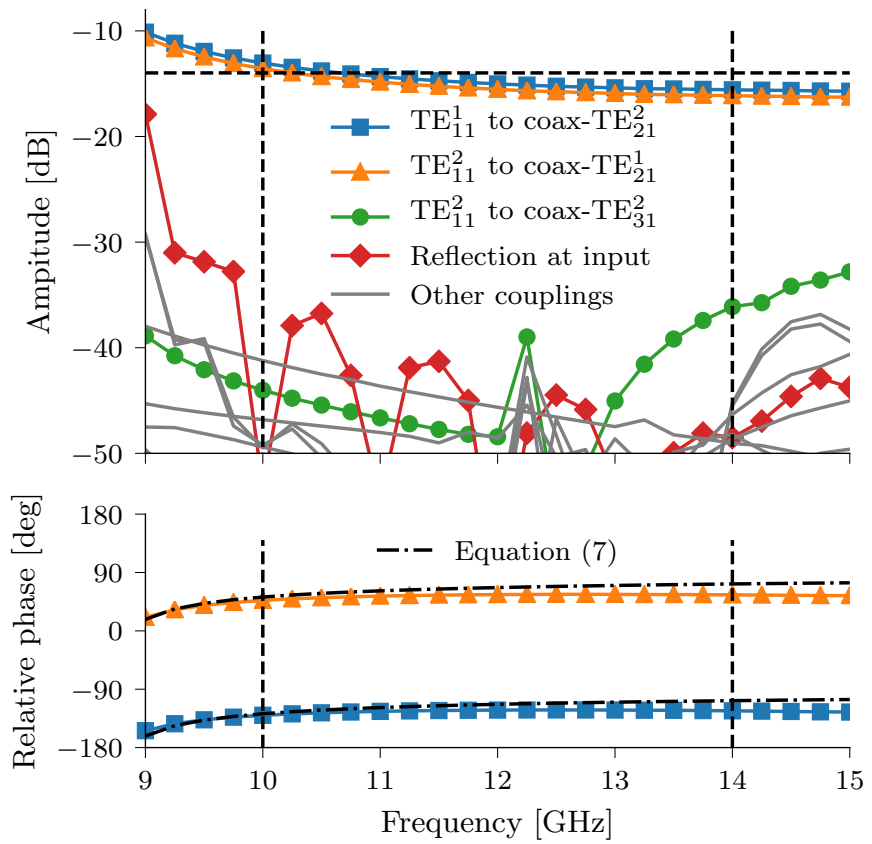

Fig. 8. Coupling from the two input modes in the circular waveguide to several modes in the coaxial guide. The two desired couplings are colored as well as the most critical undesired coupling. In addition, the maximum reflection at the input circular port is plotted in red. The horizontal dashed line indicates the desired coupling level $-14 \mathrm{~dB}$. Vertical dashed lines delimit the frequency range of interest. The phases of desired modes are plotted relative to the phase of $\mathrm{TE}_{11}$ propagation through the circular waveguide.

higher-order mode couples more than $-40 \mathrm{~dB}$ at the higher end of the band, but not to a critical degree. The two desired couplings have the same strength to within $0.5 \mathrm{~dB}$ over the entire band. The coupler is intended to be placed before a matched-feed horn antenna, feeding an offset reflector. The higher-order $\mathrm{TE}_{21}$ modes will allow suppression of cross polarization over a wide bandwidth in dual polarized operation.

\section{REFERENCES}

[1] A. W. Rudge and N. A. Adatia, "Offset-Parabolic-Reflector Antennas: A Review," Proceedings of the IEEE, vol. 66, no. 12, pp. 1592-1618, Dec. 1978.

[2] _ _ "New class of primary-feed antennas for use with offset parabolicreflector antennas," Electronics Letters, vol. 11, no. 24, pp. 597-599, Nov. 1975.

[3] K. Bahadori and Y. Rahmat-Samii, "Back-to-Back Reflector Antennas With Reduced Moment of Inertia for Spacecraft Spinning Platforms," IEEE Transactions on Antennas and Propagation, vol. 55, no. 10, pp. 2654-2661, Oct. 2007.

[4] _ - "Tri-Mode Horn Feeds Revisited: Cross-Pol Reduction in Compact Offset Reflector Antennas," IEEE Transactions on Antennas and Propagation, vol. 57, no. 9, pp. 2771-2775, Sep. 2009.

[5] Z. Pour and L. Shafai, "A Novel Dual-Mode Dual-Polarized Circular Waveguide Feed Excited by Concentrically Shorted Ring Patches," IEEE Transactions on Antennas and Propagation, vol. 61, no. 10, pp. $4917-$ 4925, Oct. 2013.

[6] _ - "Analytical Models of Dual-Polarized Primary Matched Feeds for Offset Reflector Antennas with Low Cross Polarization Properties at both Asymmetry and Diagonal Planes," IEEE Transactions on Antennas and Propagation, vol. 64, no. 5, pp. 1627-1633, May 2016.

[7] R. Jana and R. Bhattacharjee, "Matched feed design employing TE01 and TM11 modes in a smooth walled rectangular waveguide for crosspolar reduction in offset reflector antenna systems," AEU - International Journal of Electronics and Communications, vol. 69, no. 6, pp. 873-877, Jan. 2015.
[8] D. Pujara and S. B. Chakrabarty, "Cancellation of High Crosspolarization of an Offset Parabolic Reflector Antenna using a Rectangular Matched Feed," IETE Journal of Research, vol. 58, no. 4, pp. 317-321, Jul. 2012.

[9] S. B. Sharma, D. Pujara, S. B. Chakrabarty, and R. Dey, "CrossPolarization Cancellation in an Offset Parabolic Reflector Antenna Using a Corrugated Matched Feed," IEEE Antennas and Wireless Propagation Letters, vol. 8, pp. 861-864, Jul. 2009.

[10] C. Yang, J. Yu, Y. Yao, X. Liu, and X. Chen, "Novel Corrugated Matched Feed for Cross-Polar Cancellation in Tri-Reflector Compact Range," IEEE Antennas and Wireless Propagation Letters, vol. 13, pp. 10031006, May 2014.

[11] R. Dey, "Wideband Corrugated Conjugate Matched Feed Horn," Electromagnetics, vol. 37, no. 3, pp. 150-161, Apr. 2017.

[12] Z. A. Pour and L. Shafai, "A Ring Choke Excited Compact DualMode Circular Waveguide Feed for Offset Reflector Antennas," IEEE Transactions on Antennas and Propagation, vol. 60, no. 6, pp. 30113015, Jun. 2012.

[13] R. Jana and R. Bhattacharjee, "A Novel Matched Feed Structure for Achieving Wide Cross-polar Bandwidth for an Offset Parabolic Reflector Antenna System," IEEE Antennas and Wireless Propagation Letters, vol. 14, pp. 1590-1593, Mar. 2015.

[14] B. K. Watson, A. W. Rudge, and N. A. Adatia, "Dual-Polarised Mode Generator for Cross-Polar Compensation in Offset Parabolic Reflector Antennas," 8th European Microwave Conference, pp. 183-187, Sep. 1978.

[15] M. F. Palvig, E. Jørgensen, P. Meincke, and O. Breinbjerg, "Optimization Procedure for Wideband Matched Feed Design," in The 10th European Conference on Antennas and Propagation (EuCAP 2016), Davos, Switzerland, Apr. 2016.

[16] R. Dey, S. B. Chakrabarty, and R. Jyoti, "Broadband Conjugate Matched Feed Horn- A Novel Concept," IEEE Antennas and Wireless Propagation Letters, vol. 15, pp. 496-499, Jul. 2015.

[17] M. F. Palvig, E. Jørgensen, P. Meincke, and O. Breinbjerg, "Metasurface Waveguides Applied to Matched Feeds for Reflector Antennas," in The 11th European Conference on Antennas and Propagation (EUCAP 2017), Paris, France, Mar. 2017, pp. 3636-3638.

[18] G. Koch, "Coaxial Feeds for High Aperture Efficiency and Low Spillover of Paraboloidal Reflector Antennas," IEEE Transactions on Antennas and Propagation, vol. 21, no. 2, pp. 164-169, Mar. 1973.

[19] H. A. Bethe, "Theory of Diffraction by Small Holes," Physical Review, vol. 66, no. 7-8, pp. 163-182, Oct. 1944.

[20] S. E. Miller, "Coupled Wave Theory and Waveguide Applications," The Bell System Technical Journal, vol. 33, no. 3, pp. 661-719, May 1954.

[21] Y. H. Choung, K. R. Goudey, and L. G. Bryans, "Theory and Design of a Ku-Band TE21-Mode Coupler," IEEE Transactions on Microwave Theory and Techniques, vol. 30, no. 11, pp. 1862-1866, Nov. 1982.

[22] Y. H. Choung, "Wideband TM01-mode travelling wave coupler," IEE Proceedings - Microwaves, Antennas and Propagation, vol. 144, no. 5, pp. 315-320, Oct. 1997.

[23] N. Marcuvitz, Waveguide handbook. Peregrinus, 1951.

[24] E. Jones, T. Oliphant, P. Peterson et al., "SciPy: Open source scientific tools for Python," 2001-.

[25] J. McClellan and T. Parks, "A Unified Approach to the Design of Optimum FIR Linear-Phase Digital Filters," IEEE Transactions on Circuit Theory, vol. 20, no. 6, pp. 697-701, Nov. 1973.

[26] R. E. Collin, Foundations for Microwave Engineering. McGraw-Hill, 1966.

[27] M. F. Palvig, O. Breinbjerg, P. Meincke, and E. Jørgensen, "Analytical Modelling of Waveguide Mode Launchers for Matched Feed Reflector Systems," in IEEE Antennas and Propagation Society International Symposium (AP-S 2016), Puerto Rico, Jun. 2016.

[28] S. B. Cohn, "Microwave Coupling by Large Apertures," Proceedings of the IRE, vol. 40, no. 6, pp. 696-699, Jun. 1952.

[29] R. Levy, "Improved Single and Multiaperature Waveguide Coupling Theory, Including Explanation of Mutual Interactions," IEEE Transactions on Microwave Theory and Techniques, vol. 28, no. 4, pp. 331-338, Apr. 1980.

[30] "GRASP Software," TICRA, Copenhagen, Denmark, www.ticra.com.

[31] "HFSS 17.1 Software," ANSYS, Inc., Canonsburg, PA, USA, www.ansys.com.

[32] H. J. Riblet and T. S. Saad, "A New Type of Waveguide Directional Coupler," Proceedings of the IRE, vol. 36, no. 1, pp. 61-64, Jan. 1948. 